\title{
Stability of vector bundles and extremal metrics
}

\section{Burns ${ }^{\star}$ and P. de Bartolomeis}

Department of Mathematics, The University of Michigan, Ann Arbor, MI 48109, USA

Istituto di Matematica Applicata, Università degli Studi di Firenze, I-50139 Firenze, Italia

It has been known for some time now that not every compact kähler manifold of positive first Chern class admits a kähler-einstein metric, or even a kähler metric of constant scalar curvature. This is due to structure theorems of Matsushima and Lichnerowicz on the algebra of holomorphic vector fields on $\boldsymbol{M}$. For a summary, cf. [1]. Such metrics are special examples of the so-called extremal metrics of Calabi, obtained by fixing the fundamental class $[\omega] \in H^{2}(M$, $\mathbb{R}$ ), and looking for critical points $g$ of the functional

$$
I(g)=\int_{M} R^{2} \mathrm{dvol}
$$

where $g$ runs over kähler metrics with the given fundamental class and the scalar curvature and volume element are computed with respect to $g$. The EulerLagrange equations for $I(\mathrm{~g})$ can be expressed as

$$
\bar{\partial}\left(\operatorname{grad}^{(1,0)}(R)\right)=0,
$$

that is, the $(1,0)$-component of the gradient of the scalar curvature is a holomorphic vector field. The problem of finding extremal metrics is quite natural but quite difficult. Extremal metrics should be easier to find than kähler-einstein metrics or metrics of constant scalar curvature. Nevertheless, Calabi has proved some (weaker) structure theorems for the algebra of holomorphic vector fields on an $M$ with an extremal kähler metric, and $M$. Levine [8] has shown that these conditions are sufficient to obstruct the existence of an extremal metric on some $M$ with the "wrong kinds" of algebras. In a different direction, Futaki has studied the very interesting interrelationship between the algebra of holomorphic vector fields and the given kähler class $[\omega]$ which was fixed in the definition above.

In this note, we give examples of ruled surfaces $M$ which have no non-trivial holomorphic vector fields, and yet which admit no extremal kähler metric in a specifically given kähler class. For such an example, an extremal metric would

\footnotetext{
* Partially supported by the National Science Foundation (USA)
} 
necessarily be a metric of constant scalar curvature, and the obstruction found here in new in that context as well. The obstruction involves the borderline semi-stability properties of hermitian vector bundles with hermite-einstein connections (cf., e.g., [7, 9]). We came across these examples as an empirical off-shoot of our work on the integrability of twistor spaces over four-manifolds (cf. [2]). We have not been able to digest a simple general principle from the calculations, but it is clear that the borderline stability properties play the key role.

Acknowledgement. The authors would like to thank E. Calabi for the interest he has shown in this work.

To construct the examples, let $C$ be a compact Riemann surface of genus $g \geqq 2$. Consider the complex surface $S_{0}=C \times \mathbb{P}^{1}$, and give $S_{0}$ the kähler metric $g_{0}$, the product of the metric of constant curvature -1 on $C$ and that of constant curvature +1 on $\mathbb{P}^{1}$. It is easy to see that this metric has scalar curvature $R \equiv 0$.

We write $S_{0}$ in terms of vector bundles over $C$ in the obvious way, namely, $S_{0}=\mathbb{P}\left(E_{0}\right)$, where $E_{0}=C \times \mathbb{C}^{2}$. We will deform $E_{0}$ in order to construct new ruled surfaces over $C$. Write $E_{0}$ as an extension of two trivial line bundles over $C$ :

$$
0 \rightarrow L_{0} \rightarrow E_{0} \rightarrow L_{0} \rightarrow 0, \quad L_{0}=C \times \mathbb{C} .
$$

Since $g$ is non-zero, one can deform $L_{0}$ slightly to a line bundle $L$ over $C$ such that $L^{\otimes 2}$ is non-trivial. Simultaneously, one can deform the trivial extension above to an extension

$$
0 \rightarrow L \rightarrow E \rightarrow L^{*} \rightarrow 0
$$

over $C$, where $L^{*}$ denotes the dual bundle of $L$. Since $g \geqq 2, H^{1}\left(C, \mathcal{O}\left(L^{\otimes 2}\right)\right)$ is non-zero, and we can assume that $\left(^{*}\right)$ doesn't split. Let $S$ be the ruled surface $\mathbb{P}(E)$ over $C$.

Since $S$ is a small, continuous perturbation of $S_{0}$, we can identify the topological cohomology groups $H^{2}\left(S_{0}, \mathbb{Z}\right)$ and $H^{2}(S, \mathbb{Z})$, and under this identification, $c_{1}\left(S_{0}\right)=c_{1}(S)$. We let $\omega_{0}$ denote the kähler form of $g_{0}$ on $S_{0}$, and note that by the stability of kähler metrics, if $L$ is close enough to $L_{0}$ in $\operatorname{Pic}(C)$ and $\left(^{*}\right)$ is close enough to the trivial extension $0 \in H^{1}\left(C, \mathcal{O}\left(L^{\otimes 2}\right)\right)$, then the class $\left[\omega_{0}\right]$ in $H^{2}\left(S_{0}, \mathbb{R}\right)=H^{2}(S, \mathbb{R})$ is again a kähler class. We are finally in a position to state our theorem.

Theorem. If $S=\mathbb{P}(E)$ is a sufficiently small perturbation of $S_{0}$ such that $\left({ }^{*}\right)$ doesn't split and $L^{\otimes 2}$ is non-trivial, then

(i) $S$ does not admit an extremal kähler metric $g$ whose kähler class $=\left[\omega_{0}\right]$ in $H^{2}(S, \mathbb{R})$;

(ii) there are no non-trivial holomorphic vector fields on $S$.

Proof. The proof is by contradiction. The proof proceeds by a succession of simple observations. We first note that it suffices to prove the theorem with statement (i) replaced by:

(i) $S$ does not admit a kähler metric of constant scalar curvature $R$ with kähler class $\left[\omega_{0}\right]$ in $H^{2}(S, \mathbb{R})$. 
Indeed, the Euler-Lagrange equation for an extremal metric is that

$$
\bar{\partial}\left(\operatorname{grad}^{(1,0)}(R)\right)=0,
$$

and thus $\operatorname{grad}^{(1,0)}(R)$ is a holomorphic vector field, and by statement (ii) of the theorem, must be zero. Hence $R$ must be constant.

Lemma 1. Let $g$ be a kähler metric on $S$ with kähler form $\omega$ and scalar curvature R. If $[\omega]=\left[\omega_{0}\right]$, and $R$ is constant, then $R \equiv 0$.

Proof. For any compact kähler manifold $M$ of constant scalar curvature, one can calculate $R$ cohomologically:

$$
\begin{aligned}
\int_{M} c_{1}(M) \wedge \omega^{n-1} & =\frac{(n-1) !}{\pi} \int_{M} R \mathrm{dvol} \\
& =\frac{R}{\pi n} \int_{M} \omega^{n},
\end{aligned}
$$

where $n=\operatorname{dim}_{\mathbb{C}} M$. For our $S$, since $[\omega]=\left[\omega_{0}\right], c_{1}(S)=c_{1}\left(S_{0}\right)$, we get that $R=R_{0}=0$.

Lemma 2. Let $g$ be a kähler metric on $S$ with $R \equiv 0$ and $[\omega]=\left[\omega_{0}\right]$. Then $g$ is conformally flat, and the universal cover $\tilde{S}$ of $S$, with the induced metric $\tilde{\mathrm{g}}$, is holomorphically isometric to $\widetilde{S}_{0}=\Delta \times \mathbb{P}^{\mathbf{1}}$, equipped with the induced product metric. Here $\Delta=$ the unit disk.

Proof. Most of this was proved in [2], but we recall briefly the argument. One denotes by $W_{+}, W_{-}$the self-dual and anti-self-dual components of the Weyl conformal curvature tensor of $g$. For a kähler surface, $R \equiv 0$ if and only if $W_{+} \equiv 0$. Furthermore, the signature $\sigma(S)$ is $S$ is given by

$$
\sigma(S)=\frac{1}{48 \pi^{2}} \int_{S}\left\{\left|W_{+}\right|^{2}-\left|W_{-}\right|^{2}\right\} \mathrm{dvol},
$$

and since $\sigma(S)=\sigma\left(S_{0}\right)=0, W_{-} \equiv 0$. Thus $g$ is conformally flat, and more precisely, due to Theorem 1 of Derdzinski [5], $g$ is locally Hermitian symmetric. A quick glance at the (topological) possibilities shows that $\tilde{S}$ must be $\Delta \times \mathbb{P}^{1}$, as claimed. The volume of $S$ and $R \equiv 0$ fix the two constants in the Hermitian symmetric metric.

At this point we conclude that $S$ is a unitary, flat $\mathbb{P}^{1}$-bundle over $C$. That is, one has a homomorphism $\rho: \Gamma \rightarrow \operatorname{PSU}(2)$, where $\Gamma=\pi_{1}(C)=\pi_{1}(S)$, and PSU(2) is the isometry group of $\mathbb{P}^{1}$. On the other hand, $S \cong \mathbb{P}(E)$, where $E$ is uniquely determined up to tensoring with a holomorphic line bundle. One thus concludes that

(a) $\rho$ lifts to a homomorphism $\tilde{\rho}: \Gamma \rightarrow \mathrm{SU}(2)$;

(b) the lifting $\tilde{\rho}$ can be chosen so that $E$ is isomorphic to the associated flat, unitary bundle $E(\tilde{\rho})$ over $C$.

(These are because $\Lambda^{2} E \cong L \otimes L^{*}$ is trivial). Thus our $E$ admits a hermitian metric with a compatible flat connection. 
Finally, we return to $\left.{ }^{*}\right)$. Since $A^{2} E \cong L \otimes L^{*}$, one has $\operatorname{deg} E=0$. Since $\operatorname{deg} L$ $=0$ as well, by the borderline case of the theorem of Kobayashi-Lübke (cf. $[7,9]), E$ must split holomorphically and metrically as a direct sum $L \oplus L^{*}$ over $C$. This contradicts the assumption that $\left({ }^{*}\right)$ doesn't split, thereby proving part (i)' of the theorem.

Part (ii) of the theorem is a standard cohomological calculation, which we include for the convenience of the reader. Let $\pi: S \rightarrow C$ be the projection, TS, $T C$ the holomorphic tangent bundles of $S, C$ respectively, and $T F$ the line bundle over $S$ of (holomorphic) tangents along the fibers of $\pi$. One has the usual exact sequence of vector bundles over $S$ :

$$
0 \rightarrow T F \rightarrow T S \rightarrow \pi^{*}(T C) \rightarrow 0 .
$$

We wish to show $H^{0}\left(S, \mathcal{O}\left(\pi^{*} T S\right)\right)=0$.

(A) $H^{0}\left(S, \mathcal{O}\left(\pi^{*} T C\right)\right) \cong H^{0}\left(S, \pi_{*}\left(\mathcal{O}\left(\pi^{*}(T C)\right)\right)\right.$

$$
\begin{aligned}
& \cong H^{\circ}(C, \mathcal{O}(T C)) \\
& =0, \text { since } g \geqq 2 .
\end{aligned}
$$

(B) As above, $H^{0}(S, \mathcal{O}(T F))=H^{0}\left(S, \pi_{*} \mathcal{O}(T F)\right)$. It is clear that $\left.\pi_{*} \mathcal{O}(T F)\right) \cong$ $\mathcal{O}(\operatorname{sl}(E))$ on $C$, where $s l(E)$ is the bundle of traceless endomorphisms of $E$. For any $\varphi \in H^{0}(C, \mathcal{O}(\operatorname{sl}(E)))$, let $\chi$ be the composition

$$
L \longrightarrow E \stackrel{\varphi}{\longrightarrow} E \longrightarrow L^{*} .
$$

Since $\chi$ is a section of $\left(L^{*}\right)^{\otimes 2}, \chi=0$, since $\operatorname{deg} L^{*}=0$, and $\left(L^{*}\right)^{\otimes 2}$ is non-trivial. Thus, every $\varphi \in H^{0}(S, \mathcal{O}(s l(E)))$ takes $L$ to itself. The restriction of $\varphi$ to $L$ must be identically zero, since otherwise the sequence $\left({ }^{*}\right)$ would split according to the eigenspaces of $\varphi$. Thus, $\varphi$ must induce the zero map on $L^{*}$ as well, since trace $(\varphi)=0$, and $\varphi$ therefore factors through $E \rightarrow L^{*}$ and has its image in $L$. But by the same argument as above, the induced homomorphism from $L$ to $L^{*}$ is trivial, since $L^{\otimes 2}$ is non-trivial and of degree 0 . Thus, $\varphi=0$, proving part (ii) of the theorem.

We conclude this note with two remarks. First, if the curve $C$ has no nontrivial automorphisms, then $S$ has no non-trivial automorphisms. Secondly, the phenomenon above is sometimes generic, in the sense that the surfaces above form an open set in moduli, e.g., if the genus $g$ of the base curve is 2 .

\section{References}

1. Bourguignon. J.-P. et al.: Première classe de Chern et courbure de Ricci: preuve de la conjecture de Calabi. Astérisque 58 (1978)

2. Burns, D., Bartolomeis, P. de: Stable harmonic maps to $\mathbb{P}^{n}$. (To appear)

3. Calabi, E.: Extremal Kähler metrics. In: Yau, S.T. (ed) Seminar on Differential Geometry (Atrl. Math. Stud. 102, pp.259-290). Princeton: Princeton University Press 1982

4. Calabi, E.: Extremal Kähler metrics, II. In: Chavel, I., Farkas, H.M. (eds.) Differential Geomeit? and Complex Analysis. Berlin Heidelberg New York: Springer 1985, pp. 95-114 
5. Derdzinski, A.: Self-dual Kähler manifolds and Einstein manifolds of dimension four. Compos. Math. 49, 405-433 (1983)

6. Futaki, A.: An obstruction to the existence of Einstein-Kähler metrics. Invent. Math. 73, 437-443 (1983)

7. Kobayashi, S.: Curvature and stability of vector bundles. Proc. Jpn. Acad. Ser. A, Math. Sci. 58, 158-162 (1982)

8. Levine, M.: A remark on extremal metrics. J. Differ. Geom. 21, 73-77 (1985)

9. Lübke, M.: Stability of Einstein-Hermitian vector bundles. Manuscr. Math. 42, 245-257 (1983)

Oblatum 12-X-1987 